\title{
Article \\ A Solution of the Interpretation Problem of Lorentz Transformations
}

\author{
Grit Kalies* \\ HTW University of Applied Sciences Dresden; 1 Friedrich-List-Platz, D-01069 Dresden, \\ kalies@htw-dresden.de \\ * Correspondence: kalies@htw-dresden.de, Tel.: +49-351-462-2552
}

\begin{abstract}
For more than one hundred years, scientists dispute the correct interpretation of Lorentz transformations within the framework of the special theory of relativity of Albert Einstein. On the one hand, the changes in length, time and mass with increasing velocity are interpreted as apparent due to the observer dependence within special relativity. On the other hand, real changes are described corresponding to the experimental evidence of mass increase in particle accelerators or of clock delay. This ambiguity is accompanied by an ongoing controversy about valid Lorentz-transformed thermodynamic quantities such as entropy, pressure and temperature. In this paper is shown that the interpretation problem of the Lorentz transformations is genuinely anchored within the postulates of special relativity and can be solved on the basis of the thermodynamic approach of matter-energy equivalence, i.e. an energetic distinction between matter and mass. It is suggested that the velocity-dependent changes in state quantities are real in each case, in full agreement with the experimental evidence.
\end{abstract}

Keywords: interpretation problem; Lorentz transformation; special relativity; thermodynamics; potential energy; space; time; entropy; non-mechanistic ether theory 


\section{Introduction}

The Lorentz transformations were developed by the Dutch physicist Hendrik Antoon Lorentz in the years 1882-1904 [1-3]. According to Lorentz and the contraction hypothesis of George Francis FitzGerald in 1889 [4], an electron that moves with higher velocity contracts in the direction of motion due to its real interaction with the ether. Before 1905, many physicists regarded Lorentz's theory and the contraction hypothesis of FitzGerald as explanation of the Michelson-Morley experiment.

In order to offer a unified description of mechanics and electrodynamics, Albert Einstein adopted the Lorentz equations into the special theory of relativity published in 1905 [5]. Special relativity (SR) contains fundamentally new postulates, e.g. the non-existence of the ether, the invariance of the velocity of light, and the relativity of time and simultaneity. According to Einstein's reinterpretation of Lorentz's equations, length and time are observer-dependent physical quantities. In this context, time is understood as strictly symmetric.

Today the special theory of relativity is scientifically accepted, regarded as experimentally confirmed and provides the basis for the Standard Model of particle physics. Nevertheless, the criticism of the theory has not been clarified since more than one hundred years [6-12]. This does not only concern the unresolved question of irreversibility [13-15] or the ongoing controversy about the right form of Lorentz-transformed thermodynamic quantities such as entropy or temperature of a moving body within relativistic thermodynamics [16-19]. It also includes logical objections and the old dispute whether the Lorentz transformations of length, mass, etc. describe real or apparent velocity-dependent effects. Already in 1978, the philosopher, mathematician and logician Paul Lorenzen concluded:

"But exactly for the Lorentz contraction and the Einstein dilation an interpretation problem arises." („,Genau für die Lorentz-Kontraktion und die Einstein-Dilatation entsteht aber ein Interpretationsproblem.“ [20] (p. 97))

In this paper, firstly is highlighted that the interpretation problem of Lorentz-transformed quantities regarding real or apparent effects still exists today and that scientists express different personal points of view (see Section 3.1.). In Section 3.2., the origin of the interpretation problem is analyzed. It is shown that the ambiguity of Lorentz transformations within special relativity is already fixed in the assumptions, i.e. genuinely anchored in the theory. In this way, a "right" or "false" interpretation of Lorentz-transformed quantities is impossible within the framework of special relativity.

Since the interpretation problem is theory-inherent, it can be clarified only by another approach. In Section 3.3., a solution is proposed how to solve the interpretation problem on the basis of matter-energy equivalence [11,12] by refraining from the postulate of complete mass-energy equivalence. This implies ether conceptions in a modified form including ideas of Hendrik Antoon Lorentz, Lord Kelvin, Henri Poincaré, Walther Nernst, Louis de Broglie, Jean-Pierre Vigier, etc. $[21,22]$ and the acceptance of irreversible processes also in quantum physics.

\section{Materials and Methods}

The theoretical work is based on a comprehensive source-critical study of physics, physics history and philosophy. Special emphasis was placed on the analysis of the original papers of Albert Einstein, Hendrik Antoon Lorentz, Henri Poincaré, Walther Nernst, Max Planck and others. In addition, contemporary interpretation variants were studied. The method of logic was applied when evaluating the physical theories and working out a proposal for the solution of the interpretation problem of the Lorentz transformations. 


\section{Results and discussion}

At first, a short overview on the interpretative variants of the Lorentz transformations in today's textbooks will be given (Section 3.1). After that, Einstein's assumptions within the special theory of relativity are presented and discussed (Section 3.2). Finally, a solution of the interpretation problem is proposed (Section 3.3).

\subsection{Interpretation variants within special relativity}

In relativistic mechanics, the total energy $E$ of a mass point or body consisting of mass points moving in empty space is described by:

$$
E(P)=\sqrt{E_{0}^{2}+c^{2} P^{2}}
$$

with the limiting cases:

$$
\begin{array}{lll}
v=0: & E=E_{0}=m c^{2} & \text { (rest energy of mass points), } \\
v=c, m=0: & E=c P & \text { (energy of photons). }
\end{array}
$$

By means of the Lorentz factor

$$
\gamma=\frac{1}{\sqrt{1-v^{2} / c^{2}}} \geq 1,
$$

Equation (1) can be alternatively written as:

$$
E=\gamma \cdot E_{0}=\gamma \cdot m c^{2}=\frac{m c^{2}}{\sqrt{1-\left(v^{2} / c^{2}\right)}} \geq E_{0} .
$$

If $\gamma$ is developed into a Taylor series, it follows:

$$
\left(1-\frac{v^{2}}{c^{2}}\right)^{-1 / 2}=1+\frac{1}{2} \frac{v^{2}}{c^{2}}+\frac{3}{8} \frac{v^{4}}{c^{4}}+\ldots
$$

and thus for the total energy $E$ ([23] (p. 39), [24] (p. 50)):

$$
E(v)=m c^{2}+m \frac{v^{2}}{2}+\frac{3}{8} m \frac{v^{4}}{c^{2}}+\ldots
$$

Up to this point the representations in the literature correspond to each other. The interpretations of equations (1)-(5) and their derivatives, however, are very different. In special relativity, state quantities such as energy, momentum, mass, length, temperature and even a notion like time are described as dependent from the point of view of uniformly moving observers (inertial systems). In the following, some interpretation examples will be given in order to illustrate the wide range of variants in the modern technical literature.

\subsubsection{Energy parts}

At first glance, equation (5) suggests that the total energy $E$ of a moving body can be divided into the two parts rest energy $E_{0}$ and kinetic energy $E_{\text {kin: }}$

$$
E=E_{0}+E_{\text {kin }}
$$

For $v \ll c$, Equation (5) turns into the representation of classical mechanics, since the $3^{\text {rd }}$, $4^{\text {th }}$ and higher terms become negligible compared to the $2^{\text {nd }}$ term, about which Einstein wrote: 
"The second term of this series corresponds to the kinetic energy of the material point in classical mechanics.” („Das zweite Glied dieser Entwicklung entspricht der kinetischen Energie des materiellen Punktes in der klassischen Mechanik." [24] (p. 50))

Most textbook authors therefore assume a simple subdivisibility of the total energy of a particle or body at any velocity $v$ (e.g. [25-27]). The rest energy $E_{0}$ of a particle or body is interpreted as Lorentz-invariant, i.e. unaffected by the velocity $v$ according to its definition as rest energy. This implies that the $3^{\text {rd }}, 4^{\text {th }}$, etc. terms in Equation (5) are assigned to $E_{\text {kin: }}$ :

“The total energy $E$ can be divided into two parts: into the so-called rest energy [...], which the particle also has for $v=0$ due to its mass, plus the kinetic energy Ekin [...]." („Die Gesamtenergie $E$ lässt sich in zwei Anteile aufspalten: In die sogenannte Ruheenergie [...], die das Teilchen auch für $v=0$ auf Grund seiner Masse hat, plus die Bewegungsenergie [...].“ [27] (p. 27))

At this point, the first problem becomes evident. A division of $E$ into parts is only possible if the energy parts are independent of each other or, more precisely, if the parts depend on different extensive state variables, which are independent of each other.

The terminus rest energy Eo has been identified by Einstein with the total amount of energy not only of a resting mass point according to the assumptions of his theory, but also of real resting particles and bodies:

"Furthermore, if we apply the last of the equations (43) to a resting point mass [...], we see that the energy $E_{0}$ of a resting body is equal to its mass.” (,Wendet man ferner die letzte der Gleichungen (43) auf einen ruhenden Massepunkt an [...], so sieht man, dass die Energie E0 eines ruhenden Körpers seiner Masse gleich ist." [24] (p. 49))

The total amount of energy $E_{0}$ of a resting body corresponds to its internal energy $U$ (e.g. [28] (p. 235) or [29] (p. 43)). Accordingly, E0 depends on many extensive state variables:

$$
E=E_{0}\left(S, V, A, n_{i}, \ldots\right)+E_{\text {kin }}(P) .
$$

The question arises, for example, whether the volume $V$ and the interface $A$ of a body are independent of the momentum $P$, i.e. on higher velocities $v$. Some textbook authors speak therefore of the general non-subdivisibility of $E$ into $E_{0}$ and $E_{\text {kin }}$ at high velocities:

"The division [...] of the energy of a system into an 'outer' or kinetic part and an 'inner' part, i.e. the split-off of the inner energy as a part of the energy of the system, is an approximation which is only true if the condition $c|P| \ll E$ is fulfilled, which is equivalent to $|v| \ll c$." (,Die Zerlegung [...] der Energie eines Systems in einen, äußeren' oder kinetischen Anteil und einen ,inneren' Anteil, also die Abspaltung der inneren Energie als Anteil der Energie des Systems, ist eine Approximation, die nur zutrifft, wenn die Bedingung $c|P| \ll E$ erfüllt ist, die gleichbedeutend ist mit $|v| \ll c . "[30]$ (p. 144))

Even for $v \ll c$, Equation (1) may well turn into the Newtonian limiting case [30] (p. 146)

$$
E=E_{0} \sqrt{1+\frac{c^{2} P^{2}}{E_{0}^{2}}} \approx E_{0}\left(1+\frac{1}{2} \cdot \frac{c^{2} P^{2}}{E_{0}^{2}}\right)=E_{0}+\frac{P^{2}}{2 E_{0} / c^{2}},
$$

but Equation (8) is not equivalent to Equation (7), since the second term on the right side also depends on $E_{0}$ and thus on $V, S, A, n_{i}, \ldots$ Only if the changes of $E_{0}$ caused by changes of $S, V, A, n_{i}, \ldots$ are very small compared to $E_{0}$ itself, Equation (8) turns into Equation (7).

Also Max Planck has pointed out that a subdivision of $E$ can only be an approximation if thermal radiation is transported in a system:

"From now on, in principle, this subdivision is no longer permitted in any case. [...] if the body is given a certain speed, the heat radiation is set in motion at the same time. For moving heat radiation, however, although its energy depends noticeably on the speed of the motion, a separation of the energy into an internal and a progressive energy is quite impossible; consequently, such a separation is also not feasible for the total energy.” („Diese Zerlegung ist von nun an, principiell genommen, in keinem einzigen Falle mehr gestattet. [...] wenn dem Körper eine gewisse Geschwindigkeit erteilt wird, so wird diese Wärmestrahlung zugleich mit in Bewegung gesetzt. Für bewegte Wärmestrahlung aber ist, obwohl deren Energie merklich von der Geschwindigkeit der Bewegung abhängt, eine Trennung der Energie in eine innere und eine fortschreitende Energie durchaus unmöglich; folglich ist eine solche Trennung auch für die Gesamtenergie nicht durchführbar." [31] (pp. 542-543)) 
When equating $E_{0}$ with the total energy of a resting body, unsolvable interpretation problems arise. On the one hand, the rest energy $E_{0}$ must be Lorentz-invariant according to its definition as a rest property. On the other hand, $E_{0}$ depends on quantities such as $V$ and $A$, which as spatial quantities cannot be Lorentz-invariant if the transformation of length is understood as being real (cf. Section 3.1.3.). The chosen way out of the dilemma is pragmatic:

"We left out the variables $L, V$ und $A$ here. The reason is that they involve relativistic complications. [...] Since $E_{0}$ is Lorentz-invariant, we have left as variables on which $E_{0}$ depends only those which are invariant to Lorentz transformations. These are the entropy $S$ and the particle numbers [...].” („,Wir haben hier die Variablen $L, V$ und $A$ weggelassen. Der Grund liegt darin, daß sie relativistisch Komplikationen mit sich bringen. [...] Da $E_{0}$ eine Lorentz-Invariante ist, haben wir als Variablen, von denen $E_{0}$ abhängt, nur diejenigen stehenlassen, die invariant sind gegen Lorentz-Transformationen. Das sind die Entropie $S$ und die Teilchenzahlen [...].“ [30] (p. 145))

\subsubsection{Mass increase}

Due to the postulated observer dependency of state quantities and the complete mass-energy equivalence assumed by Einstein:

"Since mass and energy are the same according to the results of the special theory of relativity" („Da Masse und Energie nach den Ergebnissen der speziellen Relativitätstheorie das Gleiche sind" [31] (pp. 241-242),

the interpretation difficulties continue for the Lorentz-transformed mass: ${ }^{1}$

$$
m(v)=\frac{m}{\sqrt{1-v^{2} / c^{2}}}=\gamma m \geq m .
$$

Within the special theory of relativity, $m$ is interpreted as the mass measured in the rest system (the rest mass), whereas $m(v)$ describes the mass of a particle or body measured by an observer in a moved inertial system. $m(v)$ is sometimes called "relativistic mass", "dynamic mass" or "effective mass" with the terms are disputed today.

Observations (measurements) from differently moved inertial systems must be relative and symmetrical. Stating that "mass as a direct measure of amount of matter must be independent of the coordinate system" ([33] (p.53), some textbooks avoid interpreting $m(v)$ as real mass increase with velocity (e.g. [26] (p. 640), [33] (p. 53)). Only the rest mass $m$ is then called mass and interpreted as Lorentz-invariant quantity:

“Finally, we consider the particle's inertial mass $m$ to be a Lorentz invariant, since only 'space and time' are subject to critical revision in special relativity, not 'matter'." („,Wir betrachten schließlich noch die träge Masse $m$ des Teilchens als Lorentz-Invariante, da nur "Raum und Zeit' in der Speziellen Relativitätstheorie einer kritischen Revision unterworfen werden, nicht dagegen ,Materie'.“ [33] (pp. 49-50))

Other textbooks openly speak of a real mass increase:

"The mass of a moving particle therefore increases with its velocity $v$. [...] The work that has to be done to accelerate a mass $m$ is thus required less and less to increase the speed $v$ with increasing speed, but more and more to increase the mass.” („Die Masse eines bewegten Teilchens nimmt daher mit seiner Geschwindigkeit $v$ zu. [...] Die Arbeit, die zur Beschleunigung einer Masse $m$ aufgewendet werden muss, wird also mit wachsender Geschwindigkeit immer weniger zur Erhöhung der Geschwindigkeit v, sondern immer mehr zur Vergrößerung der Masse benötigt.“ [27] (p. 130))

Still other textbooks do not commit themselves and offer both possibilities of interpretation:

"This equation makes clear that the mass of a body increases when its speed increases. [...] But if $u$ is of the order of $c$, the body behaves as if it would have a greater mass.” („Diese Gleichung macht deutlich, daß die Masse eines Körpers anwächst, wenn sich seine Geschwindigkeit erhöht. [...] Wenn $u$ aber in die Größenordnung von $c$ kommt, verhält der Körper sich so, als ob er eine größere Masse hätte." [34] (p. 1176))

"The second equation is sometimes interpreted as if there were a relativistic mass.” („Die zweite Gleichung wird gelegentlich so interpretiert, als ob es eine relativistische Masse gäbe." [35] (p. 55))

1 Equation (9) is mostly used. Actually, there exist different equations for the $m(v)$ (see Section 3.3.2.). 
The dilemma is: If the increase in mass is real, the relativity and symmetry of observations from inertial systems (in accordance with the contents of kinematics) cannot be explained. However, if the increase in mass is not real, but only apparent, the measured real mass increases in particle accelerators cannot be explained.

Despite these contradictions, mass-increase experiments at CERN, etc. are cited in textbooks as confirmation for the postulates of relativity:

"All these findings correspond exactly to one of the most important postulates of the theory of relativity [...]." (,Alle diese Befunde entsprechen genau einem der wichtigsten Postulate der Relativitätstheorie [...]." [26] (p. 458))

\subsubsection{Length contraction}

Lorentz's equation for transforming the length $l$ of an object in the direction of motion is given by:

$$
l(v)=\sqrt{1-v^{2} / c^{2}} \cdot l=\frac{l}{\gamma} \leq l .
$$

In special relativity, Equation (10) describes measurements in different inertial systems. According to this, $l$ is the length of the rod in the resting system, which, observed from an inertial system moving against it, is shortened to $l(v)$. The rest length $l$ is thus always the greatest measured length of the rod. The contraction occurs only in the direction of motion.

Some textbook authors extend Equation (10) to the volume $V$, "since dimensions of a body perpendicular to the direction of motion are not changed" („da Abmessungen eines Körpers senkrecht zur Bewegungsrichtung nicht verändert werden“ [36] (p. 33)):

$$
V(v)=\sqrt{1-v^{2} / c^{2}} \cdot V=\frac{V}{\gamma} \leq V .
$$

Since observations (measurements) in different inertial systems are considered, they must be symmetrical due to the principle of relativity:

"Each of the observers must find the other's rod shortened by exactly the same factor against his own." (,Jeder der Beobachter muss den Stab des anderen um genau den gleichen Faktor gegen seinen eigenen verkürzt finden." [26] (p. 626))

“This reduction in length is really relative [...]. For $O, L_{2}$ appears to be shorter than $L_{1}$, whereas for $O^{\prime}, L_{1}$ appears to be shorter than $L_{2}$, i.e. the Lorentz contraction is symmetrical.” („Diese Längenverkürzung ist wirklich relativ [...]. Für $O$ erscheint $L_{2}$ kürzer als $L_{1}$ zu sein, für $O^{\prime}$ hingegen erscheint $L_{1}$ kürzer als $L_{2}$, d. h., die Lorentz-Kontraktion ist symmetrisch." [27] (p. 102)

Accordingly, length contraction is described in textbooks mostly as apparent:

"The length of a moving scale appears to the resting observer to be shorter than when the same scale is at rest relative to him.” („Die Länge eines bewegten Maßstabes erscheint dem ruhenden Beobachter kürzer zu sein, als wenn derselbe Maßstab relativ zu ihm ruhte." [27] (p. 102)

"A rod of length $l$ resting in $\Sigma$ appears in $\Sigma^{\prime}$ shopfenegb byx the factor $\left(1-\beta^{2}\right)^{1 / 2}<1$." (,Ein in $\Sigma$ ruhender Stab der Länge $l$ erscheint in $\Sigma^{\prime}$ um den Faktor

However, there are exceptions such as the textbooks of Helmut Günther [37,38], in which explicitly a real change in length of moving rods is described. Günther emphasizes the

“physical postulates of relativistic space-time” („physikalischen Postulate der relativistischen Raum-Zeit" [38] (p. 33)).

In other textbooks, the length contraction is not explicitly marked as apparent or real:

"The length of the rod is therefore smaller if it is measured in a reference system in which the rod moves." („Die Länge des Stabs ist also kleiner, wenn sie in einem Bezugssystem gemessen wird, in dem sich der Stab bewegt." [34] (p. 1162))

The dilemma is: If the increase in length is real, the relativity and symmetry of observations from inertial systems cannot be explained. Additionally, the rest energy $E_{0}=U=f(V)$ would depend 
on the velocity $v$, what it should not do as a Lorentz-invariant rest property. However, if the increase in length is not real, but only apparent, the physical postulates of relativistic spacetime, i.e. the "physical interpretation of the distance" according to Einstein ([23, p. 9]) are not fulfilled.

\subsubsection{Time dilation}

Lorentz's equation for transforming time intervals of a moved object is given by:

$$
\mathrm{d} t=\frac{\mathrm{d} \tau}{\sqrt{1-v^{2} / c^{2}}}=\gamma \mathrm{d} \tau \geq \mathrm{d} \tau .
$$

In special relativity, Equation (12) describes measurements in different inertial systems. $\mathrm{d} \tau$ is interpreted as the time interval between events that occur in the rest system, where $\tau$ is the so-called proper time. $\mathrm{d} t$ is the time interval between the same events, observed from a different moving inertial system. Two rest-system signals in the time interval $\Delta \tau=\tau_{1}-\tau_{2}$ reach the moving observer in the larger time interval $\Delta t=\gamma \Delta \tau$.

Since observations (measurements) in different inertial systems are considered, they must be symmetrical due to the relativity postulate:

"Each of the observers sees the clock of the other, moving observer running slower than his own." (,J)eder der Beobachter sieht die Uhr des anderen, bewegten Beobachters langsamer laufen als seine eigene." [26] (p. 622))

"Each oscillating system has the highest frequency in its own resting system." ("Jedes schwingende System hat in seinem eigenen Ruhsystem die größte Frequenz." [29] (p. 326))

The above two quotations agree in the statement of symmetry. However, there is a first shift in meaning because the first one speaks of an observation effect, the second one of a real change in frequencies.

In most textbooks, the slower running of moving clocks is described as real:

“Moving clocks go slower - the time dilation” („Bewegte Uhren gehen langsamer - die Zeitdilatation“ [26] (p. 620))

“Moving clocks run slower.” („Bewegte Uhren laufen langsamer.“ [27], (p. 103))

Sometimes the changes are described as apparent, as for example by the Nobel Prize laureate in physics Anthony J. Leggett:

"[...] that a physical phenomenon such as particle decay, which occurs at a certain rate when the particle is at rest, appears to an observer, relative to which the particle is moving, to occur at a reduced rate." (,, [...] dass ein physikalisches Phänomen wie der Teilchenzerfall, der, wenn das Teilchen ruht, mit einer bestimmten Rate erfolgt, einem Beobachter, relativ zu dem sich das Teilchen bewegt, mit einer reduzierten Rate vonstatten zu gehen scheint." [39] (p. 35))

While the contraction of length with speed is mostly interpreted as apparent, the delayed rate of a clock is mostly interpreted as real, although both are united in an analogous formula system. In addition, the clock delay is often referred to as time dilation - a second shift in meaning (cf. Section 3.2.).

The dilemma concerning reality or appearance of the clock-delay is the following: If the clock delay, e.g. a frequency change, is real, the relativity and symmetry of observations from inertial systems cannot be explained. Additionally, the rest energy $E_{0}=U$ would depend on the velocity $v$, which it should not do as Lorentz-invariant rest property. However, if the clock delay is not real, the real measured clock delays with higher velocity cannot be explained.

Despite this interpretation problem, the rate changes of cesium atomic clocks on board a commercial airliner [40], the delay of muon aging [41], the Ives-Stilwell experiments and many other empirical facts are interpreted as confirmation of time dilation. 


\subsubsection{Changes of thermodynamic quantities}

Equations for Lorentz-transformed thermodynamic quantities are rarely found in textbooks. In more than hundred years, neither scientific consensus on the reality or appearance of the effects was reached nor on the form of the transformation equations itself:

"We discussed the main physical ideas behind the different proposed transformations for thermodynamic quantities, with an emphasis on the temperature transformation, which has been the most controversial issue in the past, as four main different results have been proposed. [...] We also looked at the current state of the discussion, and notice that this research topic is far from being closed." [18] (p. 8)

If the rest energy $E_{0}$ of special relativity is identified with the internal energy $U$ of a system (body), the temperature $T$ can be expressed as dependence of $E_{0}$ on the entropy $S$ by keeping all other extensive state variables $X_{i}$ constant:

$$
T=\left(\frac{\partial E_{0}}{\partial S}\right)_{X_{1}, \ldots, X_{i}(\neq S)} .
$$

Analogously, the dependence of the total energy $E$ of a moving system on the entropy $S$ is:

$$
T(v)=\left(\frac{\partial E}{\partial S}\right)_{X_{1}, \ldots, X_{k}(\neq S)} .
$$

With Equation (3) for $E$ follows (e.g. [16-18,30]):

$$
T(v)=\frac{1}{\sqrt{1-\left(v^{2} / c^{2}\right)}} \frac{\partial E_{0}}{\partial S}=\frac{1}{\sqrt{1-\left(v^{2} / c^{2}\right)}} T=\gamma T \geq T
$$

with the velocity-dependent relativistic temperature $T(v)$ and the rest temperature $T$.

Since observations in different inertial systems are considered, they must be symmetrical and relative. The dilemma is again: A real change of $T$ contradicts the kinematic character of observations from inertial systems. An apparent change of $T$, however, conflicts with a real frequency change in a moved system, i.e. a measured clock delay at higher velocity.

Since 1963, Equation (15) has temporarily been predominant in the technical literature. Before 1963, Planck and Einstein had suggested that a system appears colder to the moving observer [31,42]:

$$
T(v)=\frac{T}{\gamma} \leq T .
$$

After 1963, Landsberg and van Kampen suggested that the system appears equally warm, i.e. $T(v)=T[43,44]$.

Planck and Einstein described the pressure $p$ and the entropy $S$ as Lorentz-invariant quantities [31, 42]:

$$
p(v)=p, \quad S(v)=S .
$$

Ott proposed in 1963 for the pressure $p(v)=\gamma^{2} p$ [45]. Furthermore, the opinions differ in how heat $Q=T \Delta S$ can be transformed from the point of view of the moved observer $[16,42,46]$. So far, none of the proposals has been accepted as correct and it is hoped that the experiment will bring the decision $[18,47]$.

As far as the entropy $S$ is concerned, the scientific community has agreed with the conclusion of Planck and Einstein that $S$ is to be understood as a Lorentz invariant according to Equation (17). Because $S$ plays a particularly important role in thermodynamics, Planck's proof of the Lorentz invariance in 1907 [31] will be presented and discussed briefly. 
Max Planck imagines a body that undergoes a reversible adiabatic process. Described is the view of two inertial systems, referred to as "non-dashed" and "dashed" on account of the differentiation by means of an apostrophe:

"If the entropy of the body for the non-dashed system is designated $S_{1}$ in the initial state and $S_{2}$ in the final state, then $S_{1}=S_{2}$ because of reversibility and adiabasia. But also for the dashed reference system the process is reversible and adiabatic, so we also have: $S_{1}{ }^{\prime}=S_{2}{ }^{\prime}$. [...] If $S_{1}{ }^{\prime}$ were not equal to $S_{1}$, but $S_{1}{ }^{\prime}>S_{1}$, this would mean: The entropy of the body is greater for the reference system for which it is in motion than for the reference system for which it is at rest. Then, according to this theorem, $S_{2}>S_{2}{ }^{\prime}$ would also have to be valid; because in the second state the body is at rest for the dashed reference system, while it is at rest for the non-dashed reference system. However, these two inequalities contradict the two equations given above. Neither can be $S_{1}{ }^{\prime}<S_{1}$; consequently is $S_{1}{ }^{\prime}=S_{1}$, and in general: $S^{\prime}=S$, [...] i.e. the entropy of the body does not depend on the choice of the reference system."

(„Bezeichnet man die Entropie des Körpers für das ungestrichene System im Anfangszustand mit $S_{1}$, im Endzustand mit $S_{2}$, so ist wegen der Reversibilität und Adiabasie $S_{1}=S_{2}$. Aber auch für das gestrichene Bezugssystem ist der Vorgang reversibel und adiabatisch, also haben wir ebenso: $S_{1}{ }^{\prime}=S_{2}{ }^{\prime}$. [...] Wäre nun $S_{1}^{\prime}$ nicht gleich $S_{1}$, sondern etwa $S_{1}{ }^{\prime}>S_{1}$, so würde das heissen: Die Entropie des Körpers ist für dasjenige Bezugsystem, für welches er in Bewegung begriffen ist, größer als für dasjenige Bezugsystem, für welches er sich in Ruhe befindet. Dann müsste nach diesem Satze auch $S_{2}>S_{2}$ ' sein; denn im zweiten Zustand ruht der Körper für das gestrichene Bezugsystem, während er für das ungestrichene Bezugsystem in Bewegung begriffen ist. Diese beiden Ungleichungen widersprechen aber den oben aufgestellten beiden Gleichungen. Ebenso wenig kann $S_{1}{ }^{\prime}<S_{1}$ sein; folglich ist $S_{1}{ }^{\prime}=S_{1}$, und allgemein: $S^{\prime}=S,[\ldots]$ d. h. die Entropie des Körpers hängt nicht von der Wahl des Bezugsystems ab." [31] (p. 552))

For the following reasons, Planck's argumentation must be regarded as untenable:

i) The conclusion $S^{\prime}=S$ is already included in the assumptions. Thus it represents a petitio principii. For reversible processes and reciprocal observer impressions, irreversibility is negated from the outset. Due to the reversibility of the adiabatic thought experiment, thermodynamically, $S$ can only be constant. This means that Planck defines $S$ a priori as being a Lorentz invariant.

ii) The claimed contradiction regarding the inequalities is only one if the observed change in entropy is real. In symmetric reciprocal observations, however, the change of a quantity has to be apparent. While on the one hand real effects are assumed in order to assert a contradiction, on the other hand the symmetry of the observations is emphasized. Dynamics (real effects) is mixed up with kinematics (apparent effects).

iii) Planck's equation (16) describes the temperature $T$ as a Lorentz-transformed quantity. If changes in temperature, frequency, mass, etc. with velocity are interpreted as real, they are unavoidably connected with an entropy production that occurs in any natural process according to the second law of thermodynamics. 


\subsubsection{Summary of the interpretation variants}

The textbooks of modern physics have in common that they accept and teach the special theory of relativity. The contents of teaching, however, are very diverse. In Table 1, different interpretation variants in standard textbook are summarized. The suggested variant of the respective book is marked by a cross, while a question mark is placed if a clear decision for one variant has been avoided.

Table 1: Reality or appearance of effects described by Lorentz transformation in different established physics textbooks.

\begin{tabular}{|c|c|c|c|c|c|c|c|c|}
\hline \multirow[b]{2}{*}{ Textbook } & \multicolumn{2}{|r|}{$\mathrm{d} t$} & \multicolumn{2}{|r|}{$l(v)$} & \multicolumn{2}{|c|}{$m(v)$} & \multicolumn{2}{|r|}{$T(v)$} \\
\hline & real & apparent & real & apparent & real & apparent & real & apparent \\
\hline $\begin{array}{l}\text { Falk \& Ruppel } \\
{[29,30]}\end{array}$ & $\mathbf{x}$ & & $?$ & $?$ & $x$ & & $\mathbf{x}$ & \\
\hline Tipler [34] & $\mathbf{x}$ & & $?$ & $?$ & $?$ & $?$ & & \\
\hline Gerthsen [26] & $x$ & & & $\mathbf{x}$ & & $\mathbf{x}$ & & \\
\hline Demtröder [27] & $x$ & & & $\mathbf{x}$ & $\mathbf{x}$ & & & \\
\hline Nolting [33] & $x$ & & & $\mathbf{x}$ & & $x$ & & \\
\hline Günther $[37,38]$ & $\mathbf{x}$ & & $\mathbf{x}$ & & $\mathbf{x}^{1}$ & & & \\
\hline
\end{tabular}

${ }^{1} \mathbf{x}$ : This interpretation is given.; ?: The author does not specify.

The interpretation problem is often not mentioned or ignored, even though contradictory facts are taught. Each author teaches his understanding, navigating between experimental findings that are interpreted as confirmation of special relativity and nuances of meaning.

The main results of section 3.1. are:

- $\quad$ The question if the total energy $E$ can be subdivided into the two parts rest energy $E_{0}$ and kinetic energy $E_{\text {kin }}$ remains unclear within special relativity.

- The „interpretation problem“ [20] concerning the reality or appearance of effects from the point of view of moving observers exists today as it did a hundred years ago.

- Thermodynamic quantities cannot be integrated into the theoretical concept of special relativity. 


\subsection{The origin of the interpretation problem}

In this section, the origin of the interpretation problem of Lorentz transformations is analyzed. It is shown that it is founded in the assumptions of special relativity (SR).

\subsubsection{The first two postulates of special relativity}

Einstein's first paper on special relativity [5] starts with two postulates:

a) "that rather for all coordinate systems to which the mechanical equations apply, the same electrodynamic and optical laws also apply” (,daß vielmehr für alle Koordinatensysteme, für welche die mechanischen Gleichungen gelten, auch die gleichen elektrodynamischen und optischen Gesetze gelten“ [5] (p. 891))

b) "that the light in empty space always propagates with a certain velocity $\mathrm{V}$ which is independent of the state of motion of the emitting body" („,daß sich das Licht im leeren Raum stets mit einer bestimmten, vom Bewegungszustande des emittierenden Körpers unabhängigen Geschwindigkeit V fortpflanze." [5] (p. 892))

a) and $b$ ), i.e. the principle of relativity and the independence of the speed of light from the state of motion of the light source, are - excluding the idea of empty space - intuitive and plausible. The first postulate extends Newton's principle of relativity to non-mechanical laws. The second one describes a known phenomenon, since also the speed of sound and other waves does not depend on the speed of the source.

\subsubsection{The postulates causing the interpretation problem}

Two pages after describing a) and b), Einstein adds:

"According to experience, we also state that the quantity [...] is a universal constant (the speed of light in empty space).” (,Wir setzen noch der Erfahrung gemäß fest, daß die Größe [...] eine universelle Konstante (die Lichtgeschwindigkeit im leeren Raume) sei." [5] (p. 894)

This postulate, the so-called principle of invariance, is understood as "starting point and keystone" (Ausgangs- und Angelpunkt" [25] (p. 223)) of SR. The light speed c is interpreted as an invariant universal constant. Every observer, no matter how fast he moves relative to light, measures the same value for $c$ in vacuum. The words "According to experience" [5] (p. 894) suggest that Einstein presupposes empirical confirmation for his assumption. The contra-intuitive feature is that the speed of photons is set absolute although the speed of each other elementary particle and object is known as relative.

The invariance of $c$ is not the only assumption of SR affecting the interpretation of Lorentz's equations. The individual assumptions are listed below; some of them briefly discussed from a methodical and thermodynamic point of view:

\section{$\alpha$. The assumption of the invariance of $c$}

Modern textbooks [25-28,33-38] emphasize that the invariance of $c$ is empirically confirmed. Here it should be noted that experiments always allow only partial statements and can be interpreted differently, whereby a predefined world view often plays a decisive role.

The empirical confirmation of the invariance of $c$ is lacking [8-11,48-50]. The Sagnac effect [51], on whose knowledge today's GPS technology is based, was measured in a large-scale experiment by Michelson and Gale, who interpreted the effect by a resting ether in their "Theory of the effect of the rotation of the earth on the velocity of light as derived on the hypothesis of a fixed ether" [52] (p. 137). It is suggested that the speed of light $c$ depends on the speed of the observer:

"However, the synchronization of the atomic clocks in GPS [...] confirms the change in the 'one-way' speed of light due to the motion of the earth relative to geostationary satellites." („Die Synchronisation der Atomuhren im GPS [...] bestätigt jedoch die Änderung der ,Ein-Weg'-Lichtgeschwindigkeit durch die Bewegung der Erde relativ zu geostationären Satelliten.“ [10] (p. 161)) 
The results of the Michelson-Morley and related experiments, the Fizeau experiment, etc. can be explained by understanding the ether as origin of matter [11]. In this theory, elementary particles are interpreted as ether excitations, an idea that has been already given by Henri Poincaré [21], Walther Nernst [53] and others. Like any other boson or any fermion, photons represent ether excitations and are subject to temporality.

\section{B. The idealization point mass}

In SR, moved points masses or rigid bodies consisting of point masses are described. To a point mass, the extensive state quantities rest mass $m$ and momentum $P$ are attributed.

Mechanical abstractions and idealizations are important in physics, but cannot suffice as a basis for a realistic theory of the world. Thermodynamically, $m$ and $P$ do not capture the total amount of energy of a real body. For a resting body, $m$ is one energy-equivalent property of matter, but not the only one. Spatial properties such as volume or interface represent independent energetic qualities. It has been shown that the mass equivalence of potential energy (positional energy) contradicts the first law of thermodynamics $[11,12]$.

\section{$\gamma$. The idealization empty space}

The idea "of empty space in which electromagnetic processes take place" ("des leeren Raumes, in welchem elektromagnetische Prozesse stattfinden" [5] (p. 892)) replaced the concept of light ether, which has been considered to be indispensable before 1905. In contrast to the vacuum of general relativity, that of special relativity is defined as matter- and field-free [23] (p. 120). No external forces act on an object that is moved uniformly and in a straight line. It applies: $E_{\mathrm{pot}}=0$.

Consistent with the assumptions of SR, the positional energy $E_{\text {pot }}$ of real particles and bodies is not captured by the energy concept of SR. Einstein's heuristic extension from the energy of point masses to that of real bodies proves to be insufficient [12]. In the context of SR, any theoretical proof of a complete mass-energy equivalence must necessarily remain a circular proof, because the conclusion of the theory is already anticipated in its assumptions (petitio principii).

\section{$\delta$. The interpretation of inertial systems as rigid bodies}

Within SR, inertial systems are defined as

"two coordinate systems, i.e. two systems of three rigid material lines perpendicular to each other starting from one point" (,zwei Koordinatensysteme, d.h. zwei Systeme von je drei von einem Punkte ausgehenden, aufeinander senkrechten starren materiellen Linien“ [5] (p. 897)).

This definition is one of the basic premises for the reinterpretation of space. Coordinate systems, which in Euclidean geometry are space for bodies, i.e. the outer framework for determining motion and expansion, themselves become rigid bodies. The inertial systems are "materialized", whereby spatial coordinates are transformed from conventional kinematic to dynamic quantities.

\section{$\varepsilon$. The interpretation of geometry as a branch of physics}

Distances are thought of as physical quantities what Einstein calls the "physical interpretation of distance" [23] (p. 9):

“The in such a way supplemented geometry is then to be treated as a branch of physics.” („Die so ergänzte Geometrie ist dann als ein Zweig der Physik zu behandeln." [23] (p. 9)

It should be noted that this interpretation is an assumption, not a conclusion as textbooks of modern physics (e.g. [33] (p. 5) sometimes suggest.

\section{$\zeta$. The construction of space from inertial systems}

Einstein interprets space as a "limited medium (container)": 
"So far, our space concept is bound to the box.” („Bisher ist unser Raumbegriff an die Schachtel gebunden." [23] (p. 109)) $)^{2}$

He thinks up a system of boxes (inertial systems) that move uniformly against each other and reduces the thickness of the box walls to zero [23] (p. 109). The „idea of the existence of an infinite number of spaces moving against each other" („Idee von der Existenz einer unendlichen Zahl von gegeneinander bewegten Räumen“) is described as „logically inevitable“ („logisch unvermeidlich“ [23] (p. 110)):

“But now you have to think that there are infinite spaces that move against each other." („Nun aber muss man denken, daß es unendlich viele Räume gibt, die gegeneinander bewegt sind.“ [23] (p. 110))

As no inertial system is preferred (principle of relativity), a space of reference systems moving against each other, which on the one hand are to be thought of as rigid material lines (assumption $\delta$ ) and whose wall thicknesses on the other hand approach zero, means the abandonment of the idea of an absolute space as preferred reference system.

\section{$\eta$. The interpretation of time as clock time.}

Einstein interprets time as clock time:

"We could, however, be content to evaluate the events in terms of time by an observer, who is located at the origin of the coordinate system together with the clock, which assigns to each light sign reaching him through the empty space the corresponding clock hand position, which indicates an event to be evaluated.” („Wir könnten uns allerdings damit begnügen, die Ereignisse dadurch zeitlich zu werten, daß ein samt der Uhr im Koordinatenursprung befindlicher Beobachter jedem von einem zu wertenden Ereignis Zeugnis gebenden, durch den leeren Raum zu ihm gelangenden Lichtzeichen die entsprechende Uhrzeigerstellung zuordnet." [5] (p. 893))

In this way, time, generally dimension for temporal extension, is understood as a dynamic, physical variable. Such as space, it is transformed from a kinematic to a dynamic quantity, from a dimension to its content with extension. Since clocks are physical objects, they are subject to dynamic changes. By identifying time with clock time, Einstein intends to abandon the concept of absolute time:

"According to all these definitions, spatial and temporal statements have a physical-real meaning, not just a fictitious one.” („Nach all diesen Festsetzungen haben räumliche und zeitliche Angaben eine physikalisch-reale, keine bloß fiktive Bedeutung." [24] (p. 32))

Here it should be noted that this interpretation is again an assumption, not a conclusion of SR as some textbooks suggest, and that dynamic changes cannot exist within SR as a result of the assumptions of mass points and empty space.

\section{$\theta$. The concept of the primacy of the observer}

SR is described as "theory of the dependence or invariance of physical statements on the reference system of the observer” („Lehre von der Abhängigkeit bzw. von der Invarianz physikalischer Aussagen vom Bezugssystem des Beobachters" [33] (p. 5)).

Observer impressions from inertial systems moving against each other are considered:

"[...] an observer, who is located at the origin of the coordinate system together with the clock [...]" ([...] ein samt der Uhr im Koordinatenursprung befindlicher Beobachter [...]" [5] (p. 893)).

Physics as a measuring science asks: What does the observer see from his point of view? It is obvious that each observer must recognize (measure) something different from his differently moving reference system because the propagation of light takes time. With kinematics, one usually converts observer impressions into each other.

2 The idea of space as a box goes back to Descartes and did not correspond to the prevailing philosophical views in Einstein's time. According to Kant, Hartmann and others, space as a category or dimension (not extension) is the prerequisite for expansion, but does not have any expansion itself (it is not a "box") and does not have a zero point like a coordinate system. 


\subsubsection{The internal inconsistency of special relativity}

In view of the broad range of interpretation and the multitude of idealizing and interwoven postulates in SR, it seems legitimate to ask: Can a decision be made regarding the reality or appearance of effects described by Lorentz transformations within SR?

Special relativity describes observer impressions from reference systems moving against each other. By the postulates No. 1, kinematic effects, i.e. apparent effects, are suggested:

- $\quad \beta$ : points masses or rigid bodies consisting of point masses,

- $\quad \gamma$ : motion in empty space,

- $\quad \zeta$ : space as a set of moved inertial systems,

- $\quad \theta$ : observer impressions.

Einstein understands his theory as kinematics:

"The theory to be developed is based - like any other electrodynamics - on the kinematics of the rigid body, since the statements of each theory concern relations between rigid bodies (coordinate systems), clocks and electromagnetic processes.” (,Die zu entwickelnde Theorie stützt sich - wie jede andere Elektrodynamik - auf die Kinematik des starren Körpers, da die Aussagen einer jeden Theorie Beziehungen zwischen starren Körpern (Koordinatensystemen), Uhren und elektromagnetischen Prozessen betreffen." [5] (p. 892)

Thus he explains the shortening of the length of a body in the direction of motion as apparent:

"While the $Y$ - and Z-dimensions of the sphere (i.e. of any rigid body of any shape) do not appear to be modified by the motion, the $X$-dimension appears shortened in the ratio $1: \sqrt{1-(v / V)^{2}}$, i.e. the stronger the greater $v$ is.” (,Während also die Y- und Z-Dimension der Kugel (also auch jedes starren Körpers von beliebiger Gestalt) durch die Bewegung nicht modifiziert erscheinen, erscheint die X-Dimension im Verhältnis $1: \sqrt{1-(v / V)^{2}}$ verkürzt, also um so stärker, je größer $v$ ist.“ [5] (p. 903))

Most textbook authors follow this argumentation (cf. Table 1). They conclude that it is logically incompatible to describe the same object as both: rigid and deformable.

However, the demands of SR go beyond pure motion geometry. Real effects are suggested by the postulates No. 2:

- $\delta$ : materialized inertial systems,

- $\quad \varepsilon$ : geometry as a branch of physics,

- $\eta$ : time as clock time and dynamic variable.

Since Einstein intends a "physical interpretation of distance" [23] (p. 9] and takes the changed light paths into account, he interprets clock time differences measured by light clocks in different inertial systems as real:

“How fast does this clock go when viewed from the resting system?” („Wie schnell geht diese Uhr, vom ruhenden System aus betrachtet?" [5] (p. 904))

The question is answered with:

"One concludes that a clock at the equator must run a very small amount slower than a clock at an earth's pole, which is of exactly the same quality and otherwise subject to the same conditions." („Man schließt daraus, daß eine am Erdäquator befindliche Unruhuhr um einen sehr kleinen Betrag langsamer laufen muß als eine genau gleich beschaffene, sonst gleichen Bedingungen unterworfene, an einem Erdpole befindliche Uhr." [5] (p. 905))

Again, most textbook authors follow Einstein's argumentation (cf. Table 1).

In view of postulates No. 1, it remains to be asked if the question "How fast does this clock go when viewed from the resting system?" can be relevant. Does the observation from a distant coordinate system change clock time in real terms? According to the contents of kinematics this is excluded. 
Authors who, like the physicist Mendel Sachs, pay particular attention to the postulates No. 1, conclude that only apparent changes are possible:

"It follows from this analysis that so long as there is no extra force entering the system, to physically act on the moving matter, but not to act on the matter in the observer's frame of reference (or vice versa), then there can be no asymmetric ageing - according to the logical structure of the theory of relativity itself!" [7] (p. 327)

Authors who, like the physicist Helmut Günther, pay particular attention to the postulates No. 2 conclude that the changes must be real. By assuming that the units of length and time are physical properties of atoms and molecules, Günther teaches a real length contraction of objects and real time dilation due to observations from moving inertial systems [37] (p. 11).

Authors who, like Herbert Dingle [6,54], Louis Essen [55,56] or Nicolai Hartmann [57], consider all assumptions reject the theory as logically inconsistent:

"Completely transparent, however, is the size of the aporias themselves - up to the self-contradiction of the statements - as well as the extremely narrow base of the starting position”. („Vollkommen durchsichtig ist indessen die Größe der Aporien selbst - bis zum Selbstwiderspruch der Aussagen -, sowie die äußerst schmale Basis der Ausgangsstellung." [57] (pp. 245-246))

At this point it should be noted that disregarding certain assumptions do not do justice to the full content of a theory. If all postulates of SR are taken into account, their contradictory character becomes evident. Just as it is logically incompatible to declare an object as both rigid and deformable, it is logically incompatible to interpret an effect as both kinematic and dynamic, apparent and real.

By evaluating the slowing of the clock speed as real, Einstein declares an observation effect to be reality. The observation has priority over objective reality. This idealistic position is also expressed in Einstein's thought experiments with moving trains. Since light takes time to propagate, the simultaneity of two distant events cannot be observed absolutely. This can be called the relativity of the detectability of simultaneity. The extension to the relativity of simultaneity itself, however, represents an unjustified shift in meaning [11,57]. In view of the structure of the assumptions of SR, the decision for real or apparent effects is arbitrary. However, if the effects described as apparent as Mendel Sachs suggests [7] (p. 327), SR would be no physical theory.

Einstein's thesis is the real change of clocks or even more: of time, a further shift in meaning. The changing clock rhythm is interpreted as time change. The clock is the measuring instrument, whereas time is the measured quantity. Also a ruler differs from the measured length. If the measuring tool changes, the measured quantity do not need to change at all.

The symmetry of observations and the simultaneous assertion of real time dilation have caused many controversies, e.g. $[6,7,50,56-63]$. The letter exchange in the journal Nature, which has been initiated by Herbert Dingle in 1956 and 1957, was ended in an emotional replica of several physicists in the 70s. Dingle "did not receive a logical answer" [7] (p. 331), and the special theory of relativity was classified as experimentally confirmed. The twin paradox was resolved by an asymmetry of the twins, since only one of the twins was really accelerated, i.e. left the inertial system. From a kinematic point of view, however, also accelerations cannot be set absolute, as many physicists such as Edouard Guillaume or Paul Langevin have pointed out (e.g. [60]). Kinematics only knows relative movements.

In order to support the invariance of $c$, Einstein defines time and length as dynamic variables and choses a real time dilation at higher speed. The following differences are negated [11]:

- the meaning difference between matter and mass [12],

- the meaning difference between the length of an object and a spatial coordinate, i.e. between extension and dimension [57],

- the meaning difference between clock rhythm and time, i.e. the display of a measuring instrument and the quantity measured,

- the meaning difference between observation and real effect, i.e. between kinematics and dynamics. 
Theory formation always starts with concepts. Einstein discusses "a ponderable material point" („ein ponderabler materieller Punkt" [5] (p. 919)). Here, the rhetorical figure of contradictio in adjecto is used because the terms material and point (i.e. without extension) are contradictory. As a consequence, matter and mass are often being used synonymously today. SR is characterized by the argumentative figure petitio principii (circulus in demonstrando) by postulating what needs to be proven.

From a historical view, the intern contradictions and ambiguous meanings have contributed to strengthening the theory, since the conceptual vagueness left much room for interpretation:

"You may look at special relativity from whichever side you like, you will find absolute nonsense. That's why it is so easy and - so difficult to disprove." („Man mag die spezielle Relativitätstheorie betrachten von welcher Seite man will, man stößt auf absoluten Widersinn. Deshalb ist sie so leicht und - so schwer zu widerlegen." [61] (p. 46))

If a theory already contains contradictory anticipations, physically irrelevant questions arise. While heavily criticized at the beginning [50], SR was welcomed by other scientists such as Hermann Minkowski or Max Planck [62,63]. In a multi-causal, not only scientific, but also social and science-political process, the theory has been accepted. The power of authority and the primacy of geometry, which was adopted euphorically by mathematicians, played a decisive role. ${ }^{3}$ Concepts were underestimated while mathematics flourished. In his New York lectures in 1909, Max Planck recommended that students should turn to mathematics alone:

"Since it is difficult, because of our previous habituation to the absolute concept of time, to protect ourselves from logical errors in the trains of thought necessary here without taking special precautions, it is best to follow the mathematical treatment." („Da es wegen unserer bisherigen Gewöhnung an den absoluten Zeitbegriff schwierig ist, bei den hier notwendigen Gedankengängen ohne besondere Vorsichtsmaßnahmen sich vor logischen Fehlern zu schützen, so halten wir uns dabei am besten an die mathematische Behandlungsweise." [63] (p. 118))

However, physics is not only mathematics:

"The correctness of the mathematical formalism is not sufficient to validate a scientific structure as coherent and free from contradiction [...]. In reality the two relativity theories are brimming with paradoxes." [8] (p. 248)

The assumptions must be realistic and logically consistent. The so-called "interpretation problem" [20] (p. 97), which is reflected in Table 1, is a euphemistic paraphrase for an irreconcilable contradiction. SR contradicts thermodynamics. SR and the spacetime concept of general relativity conflict with quantum mechanics. None of the postulates $\boldsymbol{\alpha}-\boldsymbol{\theta}$ helps to describe reality, and consistency is a scientific value. The experimental facts are: real mass increases of elementary particles and real clock delays with increasing speed. They can only be described and explained if the Lorentz transformations describe changes of moving objects in the non-empty space.

3 A more detailed presentation of the acceptance history of the theories of relativity is given in the textbook "Vom Energieinhalt ruhender Körper" ("From the Energy Content of Resting Bodies") [11]. 


\subsection{Return to a materialistic interpretation}

In the following, an interpretation of the Lorentz transformations is proposed, which avoids the contradictions of special relativity and explains the velocity-dependent effects by physical interactions. The basis for this is the energetic distinction between matter and mass [12] by abandoning idealizations such as point masses and empty space.

From the thermodynamic point of view, spatial properties of a system such as volume or interface, height in a gravitational field, etc. do not influence the mass, but the energy of a system. Volume and interface possess its own energetic quality. Since potential energies are not mass-relevant, mass do not represent a second energetic sum parameter of a resting body besides the internal energy $U$. Accordingly, $U$ of a resting system such as a body that represents matter is given by two energy parts [11,12]

$$
U=E_{0}+E_{\mathrm{pot}}=m c^{2}+E_{\mathrm{pot}}
$$

with the mass-equivalent, quantizable part $E_{0}=m c^{2}$ and the non-weighable, non-quantizable potential energy $E_{\text {pot. }}$

The energy part $E_{0}$ of a resting system was identified with the sum of the excitation energies of all "elementary particles" in the system. This includes:

- the intrinsic motion energies of all fermions and bosons, i.e. the internal degrees of freedom of motion, and

- the kinetic energy of all "elementary particles" and its combinations, which are moved as a whole, i.e. the external degrees of freedom of motion.

"Elementary particles" are called quantons Q [64] because they are created by excitation of a medium from which they are not independent. The former rest mass of an elementary particle is called the intrinsic mass mQ of a quanton $\mathrm{Q}$. By granting to each quanton a spatial area, the mass-equivalent intrinsic energy EQ of a quanton (fermion or boson) is:

$$
E_{\mathrm{Q}}=m_{\mathrm{Q}} c^{2}
$$

In this way, each kind of mass is interpreted as equivalent to the quantity of excitation energy, i.e. motion energy, and the equivalence of heavy and inert mass is explained. ${ }^{4}$

Equation (18) provides the theoretical justification for a non-mechanistic ether theory and a fundamental explanation of the time arrow [65]. The non-weighable, non-quantizable energy part $E_{\text {pot }}$ can only be explained by the idea of an ether or sub-quantic medium as already suggested by Oliver Heaviside, Henri Poincaré [21], Hendrik Antoon Lorentz [1-3,66,67], Louis de Broglie and Jean-Pierre Vigier [22], Walther Nernst [53] and many others.

$E_{\text {pot }}$ is identified with the positional energy of a quanton or an object in the ether medium with all neighboring excitations in continuous space and time. The ether is thought to be the origin of matter. Quantons possess both the mass-equivalent energy $E_{Q}$ and a measurable part of the non-gravitating potential ether energy. The ether medium represents energy, which does not weight itself, but causes mass. ${ }^{5}$

As ether medium, a dense and tensioned Bose-Einstein condensate was suggested that can be described with a single macroscopic wave function and serve as an absolute reference system [65].

\footnotetext{
4 Today the energy gain in nuclear reactions under mass loss is interpreted as confirmation of the complete mass-energy equivalence. This interpretation is a result of neglecting the thermodynamic method, since process conditions for nuclear reactions are not specified [11,12]. According to matter-energy equivalence, the so-called mass defect when forming a chemical bond or during nuclear reactions with release of electromagnetic energy (gamma radiation, etc.) is caused by a loss of excitation energy, i.e. motion energy.

5 In this context, ideas such as the Higgs field or quantum fluctuations of vacuum with the formation of virtual particles from nothing must be evaluated as ether substitute conceptions [11].
} 
Since quantons or its combinations can be experienced as more or less independent objects, they must be different from the unexcited ether medium, as already Hendrik Antoon Lorentz suggested:

"[...] the ether [...] with a certain degree of substantiality, however different it may be from all ordinary matter." [66] (p. 230)

Still in 1909, Lorentz defended his materialistic, non-geometric way to describe the physical behavior of a moving electron in non-empty space as the more natural one:

"In this line of thought, it seems natural not to assume at starting that it can never make any difference whether a body moves through the ether or not, and to measure distances and lengths of time by means of rods and clocks having a fixed position relatively to the ether." [66] (p. 230)

With equation (18), Henri Poincaré's wish for modification of Lorentz's ether theory can be fulfilled:

“[...]; this lightness sufficiently proves that Lorentz's theory is not an artificial construction intended to be destroyed. One must probably modify it, but one does not need to destroy it." (,[...]; diese Leichtigkeit beweist zur Genüge, daß die Lorentzsche Theorie kein künstlicher, zur Auflösung bestimmter Bau ist. Man muß sie vermutlich modifizieren, aber man braucht sie nicht zu zerstören.“ [21] (p. 176))

\subsubsection{Energy parts}

Due to the inequality $U>E_{0}$, Equation (3) for the total energy $E(P)$ of a moved point mass represents an idealization which does not include positional energies. The higher kinetic energy of an accelerated object (quanton or body) affects both its positional energy in the ether medium and its internal kinetic energies.

Increasing velocity means: An ether-dependent object is accelerated. With increasing $v$, the resistance of the ether medium against its own excitations increases, by what the mass increases in real terms (see next section). In order to further accelerate an object, one has to add ever increasing amounts of energy. In addition, the moving object is variable in its boundaries, volumes, etc. The higher the speed, the more the object is deformed in real terms. Volume work is done (see Section 3.3.3.), also interface work, electrical and magnetic work, lifting work due to the changed position in space, etc., because during the motion of the object, its neighboring excitations changes.

All kinds of energy are affected by a higher velocity $v$ of a system. The question if $E$ can be divided into two parts, which could not be answered in the context of SR, can now be answered. The two parts $U$ and $E_{k i n}$ in the formal subdivision of the velocity-dependent total energy $E$ of a macroscopic system with $i$ quantons:

$$
E(P)=U\left(P_{i}, V, A, \ldots\right)+E_{\text {kin }}(P),
$$

are not independent of each other. The velocity $v$ of the system (body, etc.) affects the velocities and momentums $P_{\mathrm{i}}$ of the quantons inside and the potential energies of the system, i.e. the volume $V$, the interface $A$, etc. since there are no rigid or impermeable "boundaries". Equation (20) represents an approximation. A subdivision into $U$ and $E_{\text {kin }}$ is only possible if the motion of a system does not noticeably influence the internal motion and the positional energy of the system. This can only be reasonably assumed if the speed of the system as a whole is negligibly small compared to that of the ether-excitations within the system.

\subsubsection{Mass increase}

If mass and the quantity of motion energy of the internal and external degrees of freedom are equivalent to each other (cf. Equation (18)), additional kinetic energy of a quanton or of a quanton cluster as a whole leads to a real increase in mass.

The problematic concept of the relativistic mass $m(v)$ within SR is often discussed today $[33,68]$. In a pragmatic way, $m(v)$ in Equation (9) is sometimes understood as a limited "recipe" [68] (p. 3). The so-called rest mass $m$ is considered as Lorentz-invariant. To this day, the textbooks differ in how 
the two force components of the Newtonian force $F=F_{N \|}+F_{N \perp}$, i.e. the part parallel and perpendicular to $v$, are treated within relativistic mechanics [68] (p. 32).

In light of matter-energy equivalence [12], the former rest mass of a quanton is caused by internal motion. With increasing velocity $v$ of a quanton or quanton cluster such as a body, the dense Bose-Einstein condensate, from which quantons emerge but are not independent, is stronger tensioned. According to this, the increase in mass with increasing $v$ is real and due to a dynamic interaction with the ether medium.

Differing from equation (9), Lorentz suggested two equations for the mass increase with velocity [67] (p. 820), the so-called longitudinal mass $m$ । when accelerating the electron in the direction of the path:

$$
m_{1}(v)=\frac{m}{1-v^{2} / c^{2 / 2}} \geq m
$$

and the transverse mass $m$ t perpendicular to it:

$$
m_{\mathrm{t}}(v)=\frac{m}{1-v^{2} / c^{2}{ }^{1 / 2}}=\gamma m \geq m .
$$

If there is no uniform rectilinear translation of the body, the equations become more difficult since angular dependencies between force and speed are taken into account. Equations (21) and (22) are today declared obsolete [68] (p. 45). However, considering that the property mass is equivalent to the quantity of excitation energy according to Equation $(18)^{6}$, the increase in mass is directional. If, like Lorentz, one assumes a strict separation of ether and electrons, then contradictions to energy conservation and to Newton's third axiom (actio est reactio) follow as Henri Poincaré highlighted [21] (p. 176). If, as is evident with matter-energy-equivalence, no strict separability of ether and matter is assumed, but quantons represent only an excited state of ether, the Lorentz theory can be further developed and the interpretation difficulties disappear.

\subsubsection{Length contraction}

In order to explain the result of the Michelson-Morley experiment [52], the Irish physicist George Francis FitzGerald (1851-1901) assumed the length contraction of a moving object in a note in Science in 1889:

"I have read with much interest Messrs. Michelson and Morley's wonderfully delicate experiment [...]. Their result seems opposed to other experiments showing that the ether in the air can be carried along only to an inappreciable extent. I would suggest that almost the only hypothesis that can reconcile this opposition is that the length of material bodies changes, according as they are moving through the ether or across it, [...]." [4] (p. 390)

Independently of him, Hendrik Antoon Lorentz explained the Michelson-Morley experiment in his physical ether theory with a length contraction of moved objects:

“The first example of this kind is MICHELSON's well known interference-experiment, the negative result of which has led FITZ GERALD and myself to the conclusion that the dimensions of solid bodies are slightly altered by their motion through the aether." [67] (p. 809)

Also a non-mechanistic quanton ether concept suggests that the described length contraction in the direction of motion [67] (p. 818) is a real deformation: ${ }^{7}$

6 Other quantizable properties such as charge, magnetic momentum or spin can be attributed to the spatial quality of ether excitation (oscillation, rotation, etc.), i.e. the quanton-internal degrees of freedom in the Euclidian space as already suggested by De Broglie and Vigier [22].

7 Based on the existing forces, Lorentz also proposes deformations in the perpendicular directions [67] (p. 818), which had to be excluded in the kinematics of special relativity. 


$$
l(v)=\sqrt{1-v^{2} / c^{2}} \cdot l=\frac{l}{\gamma} \leq l .
$$

The higher the velocity $v$, the more a body or an electron is deformed in real terms due to the increasing resistance of the ether medium to its own excitations. In the direction of motion, volume work is done as already Henri Poincaré suggested:

"We must therefore return to Lorentz's theory; but if we want to preserve it and avoid intolerable contradictions, we must assume a special force that explains both the contraction and the constancy of two axes. I have tried to determine this force and found that it is comparable to a constant external pressure acting on the deformable and compressible electron, whose work is proportional to the change in volume of the electron." ("Il faut donc en revenir à la théorie de Lorentz ; mais si l'on veut la conserver et éviter d'intolérables contradictions, il faut supposer une force spéciale qui explique à la fois la contraction et la constance de deux des axes. J'ai cherché à déterminer cette force, j'ai trouvé qu'elle peut être assimilée à une pression extérieure constante, agissant sur l'électron déformable et compressible, et dont le travail est proportionnel aux variations du volume de cet électron. » [69] (p. 130))

\subsubsection{Clock changes}

From the thermodynamic point of view, the measured changes of atomic clocks with higher velocity or gravity can be understood as real dynamic effects. A process dilatation is described. In this context, time is understood as absolute and non-symmetric, i.e. all processes become irreversible [65]. With the acceleration of quantons or quanton clusters as ether excitations, the time scale of an atomic oscillation transition is changed:

$$
\mathrm{d} t=\frac{\mathrm{d} \tau}{\sqrt{1-v^{2} / c^{2}}}=\gamma \mathrm{d} \tau \geq \mathrm{d} \tau .
$$

At higher velocities, there is a retardation of rhythmic processes. The "clock" (matter) is changed due to the increased potential energy and the higher resistance of the ether medium to its own excitations. A rhythmic process slows down under higher strain. This interpretation, which was also prioritized by philosophers and protophysicists, concedes the primacy of matter over geometry:

"The Lorentz contractions or Einstein dilatations resulting from Lorentz metrics, on the other hand, can be interpreted - as in the case of Lorentz - as shortening of bodies or slowing down of movements. One does not need to speak of a revision of space and time.” („Die Lorentzkontraktionen bzw. die Einsteindilatationen, die sich aus der Lorentzmetrik ergeben, können dagegen - wie schon bei Lorentz als Verkürzungen von Körpern bzw. Verlangsamung von Bewegungen interpretiert werden. Man brauchte nicht von einer Revision von Raum und Zeit zu sprechen.“ [70] (p. 7))

In the beginning, Lorentz did not give his time variable local time a real meaning. Thus he conceded Einstein who proposed a physical interpretation the better intuition. ${ }^{8}$ Lorentz regretted his failure already in 1909:

"The chief cause of my failure was my clinging to the idea that the variable $t$ only can be considered as the true time and that my local time $t^{\prime}$ must be regarded as no more than an auxiliary mathematical quantity." [66] (p. 321)

The rate changes of cesium atomic clocks in the Hafele-Keating experiment [40], the prolonged lifetime of muons [41], etc. can be interpreted as empirical confirmation that periodical physical processes such as atomic oscillation transitions can be influenced by $v$. In the Ives-Stilwell experiment [48], the transversal Doppler effect was demonstrated, which can be attributed to a slowing down of oscillations in the electron shells of moved ions with $v$. In modern Ives-Stilwell-like experiments, monovalent lithium ions are accelerated to 3 or 6.4 percent of the light velocity $c$ [72]. With increasing velocity of a $\mathrm{Li}^{+}$ion, the resistance of ether increases. More and more work (volume work, deformation work, etc.) is performed at the $\mathrm{Li}^{+}$ion. The increased potential energy in the $\mathrm{Li}^{+}$

8 The historical parallel in Einstein's 1905 papers is: Already in his first paper on the light-quantum hypothesis [71], he interpreted the mathematical auxiliary quantity $h$ in Max Planck's radiation law in a physical way. In his second paper, he gave physical reality to the local time of the Lorentz theory. In this case, however, Einstein did not leave it at that, but also developed a completely new theory with new assumptions about space and time and the invariance of $c$. 
quanton cluster decelerates the oscillations in the electron shell. This is connected with a lower frequency of the emitted light, which thus becomes longer wavelength (redshift). ${ }^{9}$

If the unit second today is arbitrarily defined as the duration of 9192631770 oscillations of a certain spectral line of the caesium isotope ${ }^{133} \mathrm{Cs}$, then by accelerating ${ }^{133} \mathrm{Cs}$, each individual oscillation transition of the spectral line of the ${ }^{133} \mathrm{Cs}$ is delayed. This represents no widening of the chosen unit, no change in time, but the chronometer is changed. In the case of quantons such as muons, the increased resistance of the ether medium slows down the internal kinetics so that the decay processes are slower in real terms. The kinetics of a reaction is influenced by forces.

\subsubsection{Changes of thermodynamic quantities}

Since the ether medium and its excitations are not independent of each other und the whole body or quanton is affected by motion in the non-empty space, all thermodynamic quantities are influenced by acceleration. A motion of a quanton or quanton cluster like a body in the ether medium represents a physical process.

Therefore, Planck's and Einstein's description of the entropy $S$ as a Lorentz-invariant quantity (cf. Equation 17) should be questioned. Matter-energy equivalence suggests that Lorentz-invariant quantities are simplifications and approximations being valid only in the special case of low speed and under the assumption of reversible processes.

As a result of $U>E_{0}$, it yields in case of the temperature $T$ :

$$
T=\left(\frac{\partial U}{\partial_{\mathrm{a}} S}\right)_{X_{1}, \ldots, X_{i}(\neq S)}=\left(\frac{\partial U}{\partial S-\partial_{\mathrm{i}} S}\right)_{X_{1}, \ldots, X_{i}(\neq S)} \neq\left(\frac{\partial E_{0}}{\partial S}\right)_{X_{1}, \ldots, X_{i}(\neq S)}
$$

Accordingly, equations (13)-(15) do not yet adequately describe reality. Because both the positional energy of an object in the ether medium and its internal motion are influenced by $v$, there are different effects to consider. On the one hand, volume work is done (the explanation of the Michelson-Morley experiment), and the mass of an object increases in real terms measurable in particle accelerators. On the other hand, the higher potential energy of a faster moved system leads to internal process dilation measurable as clock delay. Here it will be necessary to weigh up the effects against each other and to develop a realistic theory of quantum thermodynamics, which also covers the aging of quantons with time [65].

If we further develop a non-mechanistic ether theory, we will be able to understand nature without logical conflicts. If things go well and we believe in logic, we will describe nature more and more adequately. But we will never be able to describe it exactly.

\footnotetext{
9 Herbert Eugene Ives was, like Albert Michelson and the creator of the atomic clock Louis Essen, an opponent of the theory of relativity for his entire life and declared to have confirmed the ether of Larmor and Lorentz with his experiment [48].
} 


\section{Conclusions}

The interpretation problem of the Lorentz transformations within the special theory of relativity still exists today. By means of an analysis of the postulates of special relativity concerning energy, time and space, it has been shown that the theory contains internal inconsistencies and is characterized by shifts in meaning.

A way was proposed how to solve the interpretation problem of the Lorentz transformations on the basis of the thermodynamically justified approach of matter-energy equivalence by means of a non-mechanistic ether concept. If objects move at higher velocity, there is a real contraction in length, a real increase in mass and a real retardation of rhythmic processes.

The materialistic interpretation of the Lorentz transformations offers a number of advantages in comparison to a geometrical interpretation:

- It is logically consistent.

- It makes realistic, intuitive assumptions.

- It deals with dynamics, whereas kinematics cannot make any energetic statements.

- It describes the real changes of matter with velocity, which are experimentally evident.

- It provides concrete and vivid explanations for the behavior of matter based on the interaction of the ether medium with its excitations.

Matter-energy equivalence suggests developing a realistic theory of quantum thermodynamics. This is only possible beyond the theory of relativity and the anti-realistic Copenhagen interpretation of quantum mechanics and includes the rehabilitation and modification of ether theories. 
Funding: This research received no external funding.

Conflicts of Interest: The author declares no conflict of interest.

\section{References}

1. Lorentz, H.A. Die relative Bewegung der Erde und des Äthers. In Abhandlungen über Theoretische Physik, B.G. Teubner: Leipzig, Germany, 1892, pp. 443-447.

2. Lorentz, H.A. Versuch einer Theorie der electrischen und optischen Erscheinungen in bewegten Körpern, E. J. Brill: Leiden, Germany, 1895.

3. Lorentz, H.A. Weiterbildung der Maxwellschen Theorie. Elektronentheorie. In Encyclopädie der mathematischen Wissenschaften mit Einschluss ihrer Anwendungen, Volume 5, Part 2, B.G. Teubner: Leipzig, Germany, 1904, pp. 145-288.

4. FitzGerald, G.F. The Ether and the Earth's Atmosphere. Science 1889, 13, p. 390.

5. Einstein, A. Zur Elektrodynamik bewegter Körper. Annalen der Physik 1905, 322, pp. 891-921.

6. Dingle, H. Science at the Crossroads, Martin Brian \& O'Keeffe: London, United Kingdom, 1972.

7. Sachs, M. On Dingle's Controversy about the Clock Paradox and the Evolution of Ideas in Science. International Journal of Theoretical Physics 1974, 10, pp. 321-331.

8. Selleri, F. Recovering the Lorentz Ether, Apeiron 2004, 11, pp. 246-281.

9. v. Mettenheim, Ch. Einstein, Popper and the Crisis of Theoretical Physics, Tredition: Hamburg, Germany, 2015.

10. Jooß, Ch. Selbstorganisation der Materie. Dialektische Entwicklungstheorie von Mikro- und Makrokosmos, Neuer Weg: Essen, Germany, 2017.

11. Kalies, G. Vom Energieinhalt ruhender Körper: Ein thermodynamisches Konzept von Materie und Zeit, De Gruyter: Berlin, Germany, 2019.

12. Kalies, G. Matter-Energy Equivalence. Zeitschrift für Physikalische Chemie 2019, DOI: 10.1515/zpch-2019-1487.

13. Straub, D. Eine Geschichte des Glasperlenspiels. Irreversibilität in der Physik: Irritationen und Folgen, Birkhäuser: Basel, 1990.

14. Smolin, L. Time Reborn: From the Crisis in Physics to the Future of the Universe, Penguin Books: United Kingdom, 2014.

15. Haddad, W.M. Thermodynamics: The Unique Universal Science. Entropy 2017, 19, 621 (pp. 1-70).

16. Neugebauer, G. Relativistische Thermodynamik, Akademie-Verlag: Berlin, Germany, 1980.

17. Wang, C.-Y.: Thermodynamics since Einstein. Advances in Natural Science 2013, 6, pp. 13-17.

18. Farías, C.; Pinto, V.A.; Moya, P.S. What is the temperature of a moving body? Scientific Reports 2017, 7, 17657 (pp. 1-9).

19. Parvan, A.S.: Lorentz transformations of the thermodynamic quantities, Annals of Physics 2019, 401, pp. $130-138$.

20. Lorenzen, P.: Theorie der technischen und politischen Vernunft, Reclam: Stuttgart, Germany, 1978.

21. Poincaré, H.: Wissenschaft und Hypothese, B.G. Teubner: Leipzig, Germany, 1904 (1 $1^{\text {st }}$ edition 1902: La science et l'hypothèse, Flammarion: Paris).

22. Broglie, L. de. Introduction à la nouvelle théorie des particules de M. Jean-Pierre Vigier et de ses collaborateurs, Gauthier-Villars : Paris, France, 1961.

23. Einstein, A. Über spezielle und allgemeine Relativitätstheorie, Akademie-Verlag: Berlin, Germany, 1969 ( $1^{\text {th }}$ edition 1917).

24. Einstein, A. Grundzüge der Relativitätstheorie, Akademie-Verlag: Berlin, Germany, 1969 (1 $1^{\text {th }}$ edition 1922).

25. Scheck, F. Theoretische Physik 1. Mechanik, Springer: Berlin, Germany, 2002.

26. Meschede, D. Gerthsen Physik, Springer: Berlin, Germany, 2005.

27. Demtröder, W. Experimentalphysik I: Mechanik und Wärme, Springer: Berlin, Germany, 2005.

28. Tipler, P.A.; Mosca, G. Physics for Scientists and Engineers, H. Freeman and Company: New York, United States, 2008.

29. Falk, G.; Ruppel, W. Mechanik Relativität Gravitation, Springer, Berlin, Germany, 1973.

30. Falk, G.; Ruppel, W. Energie und Entropie, Springer, Berlin, Germany, 1976.

31. Planck, M.: Zur Dynamik bewegter Systeme. Sitzungsberichte der Königlich Preußischen Akademie der Wissenschaften zu Berlin 1907, 1, pp. 542-570.

32. Einstein, A.: Prinzipielles zur allgemeinen Relativitätstheorie. Annalen der Physik 1918, 55, pp. 241-244. 
33. Nolting, W. Grundkurs Theoretische Physik 4. Spezielle Relativitätstheorie. Thermodynamik, Springer: Berlin, Germany, 2010.

34. Tipler, P.A. Physik, Spektrum Akademischer Verlag: Heidelberg, Germany, 2000.

35. Kurzweil, P.; Frenzel, B.; Gebhard, F. Physik Formelsammlung. Für Ingenieure und Naturwissenschaftler, Vieweg \& Teubner: Wiesbaden, Germany, 2008.

36. Meinel, R. Spezielle und allgemeine Relativitätstheorie für Bachelorstudenten, Springer: Berlin Heidelberg, Germany, 2016.

37. Günther, H. Starthilfe Relativitätstheorie. Ein neuer Einstieg in Einsteins Welt, Vieweg \& Teubner: Wiesbaden, Germany, 2010.

38. Günther, H. Die Spezielle Relativitätstheorie: Einsteins Welt in einer neuen Axiomatik, Springer Spektrum: Wiesbaden, Germany, 2013.

39. Leggett, A.J. Physik: Probleme-Themen-Fragen, Birkhäuser: Basel, Switzerland, 1989.

40. Hafele, J.C.; Keating, R.E. Around-the-world atomic clocks: predicted relativistic time gains. Science 1972, 177, pp. 166-168.

41. Rossi, B.; Hall, D.B.: Variation of the Rate of Decay of Mesotrons with Momentum. Physical Review 1941, 59, pp. 223-228.

42. Einstein, A.: Über das Relativitätsprinzip und die aus demselben gezogenen Folgerungen. Jahrbuch der Radioaktivität und Elektronik 1908, 4, pp. 411-462.

43. Landsberg, P.T. Does a Moving Body Appear Cool? Nature 1966, 212, pp. 571-572.

44. van Kampen, N.G. Relativistic Thermodynamics of Moving Systems. Physical Review 1968, 173, pp. 295301

45. Ott, H.: Lorentz-Transformation der Wärme und der Temperatur. Zeitschrift für Physik 1963, 175, pp. 70104.

46. Callen, H.; Horwitz, G. Relativistic Thermodynamics. American Journal of Physics 1971, 39, pp. $938-947$.

47. Krizan, J.E. Temperature and relativistic thermodynamics. Physics Letters 1979, 71, pp. 174-176.

48. Ives, H.E.; Stilwell, G.R: An Experimental Study of the Rate of a Moving Atomic Clock. Journal of the Optical Society of America 1938, 28, pp. 215-226.

49. Hazelett, R.; Turner, D. (Ed.): The Einstein Myth and the Ives Papers: A Counter-Revolution in Physics, Devin-Adair: Old Greenwich, United States, 1979.

50. Mueller, G.O. (Ed.): Über die absolute Größe der Speziellen Relativitätstheorie. Ein dokumentarisches Gedankenexperiment über 95 Jahre Kritik (1908-2003) mit Nachweis von 3789 kritischen Arbeiten, available from: https://www.ekkehard-friebe.de/buch.pdf, [cited July 27, 2020].

51. Sagnac, G. Sur la preuve de la réalité de l'éther lumineux par l'expérience de l'interférographe tournant. Comptes Rendus de l'Académie des Sciences 1913, 157, pp. 1410-1413.

52. Michelson, A.A., Gale, H.G. The Effect of the Earth's Rotation on the Velocity of Light. Astrophysical Journal 1925, 61, pp. 140-145.

53. Nernst, W.: Über einen Versuch, von quantentheoretischen Betrachtungen zur Annahme stetiger Energieänderungen zurückzukehren (Lecture from 18.01.1916). Verhandlungen der Deutschen Physikalischen Gesellschaft 1916, 18, pp. 83-116.

54. Dingle, H.: The Case against Special Relativity. Nature 1967, 216, pp. 119-122.

55. Essen, L. The Clock Paradox of Relativity. Letters to Nature 1957, 180, pp. 1061-1062.

56. Essen, L.: Relativity - joke or swindel? Electronics \& Wireless World 1988, 94, pp. 126-127.

57. Hartmann, N.: Philosophie der Natur, Walther de Gruyter: Berlin, Germany, 1950.

58. Bergson, H. Durée et simultanéité. À propos de la théorie d'Einstein, Félix Alcan : Paris, France, 1922.

59. Galeczki, G., Marquardt, P. Requiem für die Spezielle Relativität, Haag \& Herchen: Frankfurt am Main, Germany, 1996.

60. Guillaume, E. Les bases de la physique moderne. Archives des sciences physiques et naturelles 1917, 43, pp. 89-112.

61. Weinmann, R. Der Widersinn und die Überflüssigkeit der speziellen Relativitätstheorie. Annalen der Philosophie und philoshischen Kritik 1929, 8, pp. 46-57.

62. Minkowski, H. Raum und Zeit, Vortrag auf der 80. Naturforscherversammlung, Köln (1908). In Jahresberichte der Deutschen Mathematiker-Vereinigung, Leipzig, Germany, 1909.

63. Planck, M. Acht Vorlesungen über theoretische Physik. Gehalten an der Columbia University in the City of New York im Frühjahr 1909, Hirzel: Leipzig, Germany, 1910. 
64. Lévy-Leblond, J.M.: Von der Materie, Merve Verlag: Berlin, Germany, 2012.

65. Kalies, G. A solution of the time paradox of physics. Zeitschrift für Physikalische Chemie, accepted with minor revision, $7 / 2020$.

66. Lorentz, H.A. The Theory of Electrons, B.G. Teubner: Leipzig, Germany, 1916 (1 $1^{\text {st }}$ edition 1909).

67. Lorentz, H.A. Electromagnetic phenomena in a system moving with any velocity smaller than that of light. In KNAW Proceedings, Amsterdam, 1903-1904; Volume 6, pp. 809-831.

68. Fließbach, T. Die relativistische Masse, Springer : Berlin, Germany, 2018.

69. Poincaré, H.: Sur la dynamique de l'électron. Rendiconti del Circolo matematico di Palermo 1906, 21, pp. 129176.

70. Lorenzen, P.: Relativistische Mechanik mit klassischer Geometrie und Kinematik. Mathematische Zeitschrift 1977,155 , pp. 1-9.

71. Einstein, A.: Über einen die Erzeugung und Verwandlung des Lichts betreffenden heuristischen Gesichtspunkt. Annalen der Physik 1905, 17, pp. 132-148.

72. Reinhardt, S.; Saathoff, G.; Buhr, H.; Carlson, L.A.; Wolf, A.; Schwalm, D.; Karpuk, S.; Novotny, C.; Huber, G.; Zimmermann, M.; Holzwarth, R.; Udem, T.; Hänsch, T.W.; Gwinner, G. Test of relativistic time dilation with fast optical atomic clocks at different velocities. Nature Physics 2007, 3, 861-864.

\begin{tabular}{lll}
\multicolumn{2}{l}{ List of symbols } & \\
$A$ & $/ \mathrm{m}^{2}$ & interface area \\
$E$ & $/ \mathrm{J}$ & total energy \\
$E_{0}$ & $/ \mathrm{J}$ & rest energy / intrinsic energy \\
$l$ & $/ \mathrm{kg}$ & length \\
$l(v)$ & $/ \mathrm{kg}$ & velocity-dependent length \\
$m$ & $/ \mathrm{kg}$ & mass \\
$m(v)$ & $/ \mathrm{kg}$ & velocity-dependent mass \\
$n$ & $/ \mathrm{mol}$ & amount of substance \\
$p$ & $/ \mathrm{J} / \mathrm{m}^{3}$ & pressure \\
$P$ & $/(\mathrm{kg} \mathrm{m})$ & momentum \\
$S$ & $/ \mathrm{J} / \mathrm{K}$ & entropy \\
$t$ & $/ \mathrm{s}$ & time \\
$\tau$ & $/ \mathrm{s}$ & local time, proper time, etc. \\
$T$ & $/ \mathrm{K}$ & absolute temperature \\
$T(v)$ & $/ \mathrm{K}$ & velocity-dependent absolute temperature \\
$U$ & $/ \mathrm{J}$ & internal energy \\
$V$ & $/ \mathrm{m}^{3}$ & volume \\
$v$ & $/ \mathrm{m} / \mathrm{s}$ & velocity \\
$\mathrm{d}_{\mathrm{i}}$ & & change by internal processes \\
$\mathrm{d}$ a & & change by exchange processes \\
& \multicolumn{1}{c}{1} & \\
$\gamma=\frac{\sqrt{1-v^{2} / c^{2}}}{21}$ & Lorentz factor \\
&
\end{tabular}

\section{Indices}

kin kinetic

pot potential

1 longitudinal

$\mathrm{t}$ transverse

Q quanton

\section{Constants}

velocity of light $c$, Avogadro number $N_{\mathrm{A}}$, Boltzmann constant $k_{\mathrm{B}}$, general gas constant $R$, Planck's constant $h$ 